\title{
A Novel Doxorubicin Prodrug with Controllable Photolysis Activation for Cancer Chemotherapy
}

\author{
Stuart Ibsen • Eran Zahavy $\cdot$ Wolf Wrasdilo • Michael Berns • Michael Chan • Sadik Esener
}

Received: 8 November 2009 / Accepted: 1 June 2010 / Published online: 2 July 2010

(C) The Author(s) 2010. This article is published with open access at Springerlink.com

\begin{abstract}
Purpose Doxorubicin (DOX) is a very effective anticancer agent. However, in its pure form, its application is limited by significant cardiotoxic side effects. The purpose of this study was to develop a controllably activatable chemotherapy prodrug of DOX created by blocking its free amine group with a biotinylated photocleavable blocking group (PCB).

Methods An n-hydroxy succunamide protecting group on the PCB allowed selective binding at the DOX active amine group.

The PCB included an ortho-nitrophenyl group for photo cleavability and a water-soluble glycol spacer arm ending in a biotin group for enhanced membrane interaction.
\end{abstract}

Results This novel DOX-PCB prodrug had a 200-fold decrease in cytotoxicity compared to free DOX and could

\section{S. Ibsen $(\bowtie)$}

Department of Bioengineering, Moores Cancer Center

University of California San Diego

3855 Health Sciences Dr. \# 08I5

La Jolla, California 92093-08I5, USA

e-mail: sibsen@ucsd.edu

\section{E. Zahavy}

Department of Physical Chemistry

Israel Institute for Biological Research

P.O. Box 19, Ness-Ziona 74100, Israel

W. Wrasdilo $\cdot$ M. Chan

Moores Cancer Center, University of California at San Diego

La Jolla, California 92093, USA

\section{Berns}

Department of Bioengineering, University of California at San Diego

La Jolla, California 92093, USA

\section{S. Esener}

Department of Nanoengineering

University of California at San Diego

La Jolla, California 92093, USA release active DOX upon exposure to UV light at $350 \mathrm{~nm}$. Unlike DOX, DOX-PCB stayed in the cell cytoplasm, did not enter the nucleus, and did not stain the exposed DNA during mitosis. Human liver microsome incubation with DOX-PCB indicated stability against liver metabolic breakdown.

Conclusions The development of the DOX-PCB prodrug demonstrates the possibility of using light as a method of prodrug activation in deep internal tissues without relying on inherent physical or biochemical differences between the tumor and healthy tissue for use as the trigger.

KEY WORDS prodrug · photocleavable · photoactivatable . doxorubicin · toxicity

\section{INTRODUCTION}

Doxorubicin (DOX) is an effective antitumor antibiotic that is used extensively in the treatment of different human cancers, including breast cancer, soft tissue sarcomas, and Hodgkin's and non-Hodgkin's lymphomas $(1,2)$. Although it is one of the more potent chemotherapy agents known, its clinical application is limited by its harmful side effects, the most significant of which is its cardiotoxicity, which can lead to cardiomyopathy and congestive heart failure (2-4).

Considerable work has been undertaken to chemically modify DOX and other chemotherapy agents with the goal of reducing their systemic toxicity. One such route is to create prodrugs which exhibit reduced toxicity to healthy tissue and can be transformed into pharmacologically active agents specifically within the tumor region. (4-6). Several designs have taken advantage of environmental differences between the tumor tissue and normal tissue, such as hypoxia or low $\mathrm{pH}$, to trigger the prodrug transformation $(7,8)$. Some prodrugs have been designed to be activated by enzymes 
that are secreted by the tumor tissue at higher levels than elsewhere in the body (9-11). The degree of tumor specificity for prodrug activation in these cases depends upon the degree of difference between the tumor tissue and the healthy tissue with respect to these parameters.

Some prodrug designs focus on tumor-specific antigens that are expressed on the surface of the tumor cells. Attaching antibodies to the chemotherapy agents that are specific for those antigens excludes the agent from nontargeted healthy cells. The transformation of the agent is triggered upon antibody/antigen binding and internalization into the cell, usually triggered by the low $\mathrm{pH}$ of the intracellular endosome environment (12). These strategies can achieve higher degrees of tumor-specific prodrug activation with increasing amounts of knowledge about the internal environment and the surface antigens specific to a certain tumor. However, tumor cells are not homogenous in their gene expression (13). This makes targeting every single cell difficult. They are also not homogenous in their physical location relative to the tumor's hypoxic or low-pH zones. Some cells can be difficult to reach, especially with bulky prodrugs, due to poor circulation networks within the tumor tissue. This requires that the prodrug be activated to a very potent drug which can affect cells in the general region of activation.

Instead of relying on inherent differences between the tumor and the healthy tissue to activate prodrugs, it is possible to modify the tumor environment and take advantage of those artificial changes to differentiate the tumor region from the rest of the body. One method is to administer therapeutic ionizing radiation and use the small amount of reducing chemical species that can be created through water lysis. These extra reducing species could theoretically fill the role of reducing enzymes in the activation of the prodrug as long as the cleavable linker is sensitive enough to respond to small amounts of the active species produced (14). Work has also been done to demonstrate the feasibility of covalently attaching photoliable blocking groups to paclitaxel with the capability of releasing paclitaxel upon light exposure (15). However, the biological consequences of attaching the blocking group and the activity of the released paclitaxel have not been studied.

Another method is to use non-human enzymes to activate the prodrug. This reduces the possibility of any undesired prodrug activation outside the desired region as long as the enzyme itself, or the gene encoding for the enzyme, can be delivered to the tumor tissue specifically $(16,17)$.

From previous work in this field, a few prodrug properties can be identified for use in maximizing the effectiveness of the prodrug. It is desirable that the prodrug be a modified version of a potent chemotherapy drug and have greatly reduced toxicity compared to the drug itself. The use of a trigger source which is external to the body and localized specifically to the tumor tissue of interest avoids relying on inherent properties of the tumor to differentiate it from normal tissue. The ability of the prodrug to enter cells and maintain its prodrug properties is important to allow time for the circulating prodrug to clear from the system. This ensures only the prodrug that entered the targeted cells is trigged by the external source. Once triggered to activate, the prodrug should release the drug in its intact and pharmacologically active form.

The aim of this study was to design a prodrug that possess these desirable properties. The final design was a caged form of DOX created by blocking its free amine group with a biotinylated photocleavable blocking group (PCB) (18). This novel DOX-PCB prodrug can be activated upon exposure to UV at $350 \mathrm{~nm}$ to release intact DOX. The photocleavable properties come from the incorporation of an o-nitrophenyl group (19). UV administered to the tissue region of choice, possibly by use of fiber optics or miniature UV light-emitting diodes, could trigger active DOX release from the DOX-PCB prodrug specifically in this region.

The introduction of UV radiation to the deep internal tissues through the use of implanted fiber optics or UV lightemitting diodes would create a significant and controllable difference between the tumor and the healthy tissue. The only significant source of UV in the deep internal tissues of the body would be the delivered UV, since only $1 \%$ of $360 \mathrm{~nm}$ UV is transmitted through $1 \mathrm{~mm}$ of human skin due to melanin content (20). The $350 \mathrm{~nm}$ light has significant penetration through tissue that does not contain melanin with minimal absorption (21). The $350 \mathrm{~nm}$ light also has minimal absorption by DNA (22). The use of UV photocleavable prodrugs, such as DOX-PCB, could allow for activation of the prodrug just in the UV-exposed deep tissue region. A higher degree of spatial and temporal control could be achieved this way rather than relying solely on inherent differences between the tumor and the healthy tissue to act as the trigger. The effective spatial range of the UV dose, and subsequent active DOX release, in the tissue can be manipulated by adjusting intensity, duration of exposure, and placement of the UV diode or fiber optic. The scattering properties of the tissue would help ensure a uniform exposure of the entire region of interest. The timing of the administration of the UV dose can also be controlled to occur when DOX-PCB reaches its most concentrated levels in the tumor region. In this continuing investigation we have demonstrated the feasibility of this approach.

\section{MATERIALS AND METHODS}

\section{Materials}

Doxorubicin hydrochloride (DOX) was obtained from Qventas (Branford, CT, USA) and Sigma (St. Louis, 
MO, USA). Water-soluble photocleavable biotin-NHS (PCB) was purchased from Ambergen (Watertown, MA, USA). The components of the synthesis buffer, $\mathrm{NaCHO}_{3}$ and $\mathrm{NaOH}$, were from EMD (San Diego, CA, USA). The NADPH regeneration system was purchased from BD Biosciences (San Jose, CA, USA). The human liver microsomes and the TOX2 In Vitro Toxicology XTT Assay Kit were both purchased from Sigma (St. Louis, MO, USA). The DMEM cell culture media and the trypsin were purchased from Mediatech Inc. (Manassas, VA, USA). The penicillin-streptomycin used in the DMEM medias, the advanced MEM Media, and the DMEM Media without phenol red were purchased from Gibco (Invitrogen, Grand Island, NY, USA). The fetal bovine serum used in the DMEM media solutions was purchased from Hyclone (Logan, UT, USA). HPLC-grade acetonitrile and methanol were purchased from Fisher Scientific (Fairlawn, NJ, USA). The methanol- $\mathrm{d}_{4}$ and deuterated dimethlyl sulfoxide $\left(\mathrm{DMSO}-\mathrm{d}_{6}\right)$ were purchased from Cambridge Isotope Laboratories Inc. (Andover, MA, USA). The preparative thin-layer silica gel liquid chromatography plates were purchased from Merck. The water used was first filtered with the Milli-Q Plus System (Millipore Corporation, Bedford, USA). The cell line used for the cytotoxicity studies was the human lung cancer cell line A549 purchased from the American Type Culture Collection (ATCG) (Manassas, VA, USA). The PTK2 (Potorous tridactylus) kidney epithelial cell line was originally purchased from the ATCG and kindly provided by the Beckman Laser Institute (UC Irvine, CA).

\section{Conjugation of DOX to PCB}

DOX was dissolved in water to a $1.2 \mu \mathrm{M}$ concentration. A $0.1 \mathrm{M} \mathrm{NaCHO}_{3} / \mathrm{NaOH}$ buffer at $\mathrm{pH} 9.3$ was added to the DOX solution to bring the total $\mathrm{pH}$ to 9.0. The DOX/ buffer solution was then added to $1 \mathrm{mg}(1.2 \mu$ moles $)$ of dry water-soluble photocleavable biotin (PCB) in a 1:1 molar ratio. The reaction was stirred vigorously with a magnetic Teflon-coated stir bar for $30 \mathrm{~min}$. The DOX-PCB prodrug product precipitated out. The nearly colorless supernatant was removed, and the precipitate was dissolved in DMSO. The synthetic scheme is shown in Fig. 1.

\section{Purification of DOX-PCB}

Purification was done by preparative thin-layer liquid chromatography using a silica gel on a small scale for initial characterization. Later, an HPLC semiprep purification was used to obtain larger amounts of DOX-PCB at a higher degree of purity for cell culture work and the DOX-PGB UV stability study. The purification of DOXPCB in DMSO was done on an Agilent 1200 Series LC
(Santa Clara, CA, USA) with a Luna 5u C-18(2) 100A column (250 $\mathrm{mm} \times 15 \mathrm{~mm}$, with a $5 \mu \mathrm{m}$ bore size) (Phenomenex, Torrance, CA, USA). Solution A was $100 \%$ water, and solution B was $100 \% \mathrm{MeOH}$. B was increased from $10-100 \%$ over 20 min with a flow rate of $50 \mu \mathrm{L} / \mathrm{min}$. Two isomeric forms of DOX-PCB were observed to separate slightly from each other on the column and were collected together in the same sample. The two isomers were also collected separately from one another, and no differences in cytotoxicity between the two forms were observed. The overall yield of pure DOX$\mathrm{PCB}$ was $70 \%$.

\section{Characterization}

The DOX-PGB compound was characterized by high resolution mass spectrometry, HPLC/mass spectrometry, and an absorption spectral analysis.

High resolution mass spectrometry was performed on a Thermo Scientific LTQ Orbitrap XL mass spectrometer with electrospray ionization source (Milford, MA, USA). The sample of purified DOX-PGB was dissolved in a mixture of DMSO/water (20/80, v/v).

The HPLC/mass spectrometry analysis was performed on a Thermo Finnigan LCQdeca mass spectrometer (Milford, MA, USA) using a Magic C-18 column $(1.0 \mathrm{~mm} \times 150 \mathrm{~mm})$ (Michrom BioResources, Auburn, CA). Solution A was 2.5\% $\mathrm{MeOH}$ in water, and Solution B was $100 \% \mathrm{MeOH}$. B was increased from $10-95 \%$ over $20 \mathrm{~min}$ and then held at $95 \%$ for $5 \mathrm{~min}$. The flow rate was $50 \mu \mathrm{L} / \mathrm{min}$. The mass spectrometry portion used electrospray ionization (ESI) in positive ion mode. The sample of purified DOX-PGB was dissolved in a mixture of DMSO/water (20/80, v/v).

An absorption spectrum was taken of a purified sample of DOX-PCB in DMSO and of DOX in DMSO using a NanoDrop ND-1000 Spectrophotometer (Wilmington, DE, USA). The measurements of DOX and DOX-PCB (data not shown) reveal that both compounds had an absorption maxima at $\lambda=485 \mathrm{~nm}$ from the DOX chromophore. DOX-PCB had an absorption at $350 \mathrm{~nm}$ due to the nitrophenyl group. This group enabled the photolysis process to occur upon absorption of radiation at $350 \mathrm{~nm}$. The DOX-PCB spectra had the following 6 peaks: UVvisible (abs. DMSO): 260, 287, 480, 500, 530, $584 \mathrm{~nm}\left(\varepsilon_{c m}^{1 \%}\right.$ : $\left.25.9,14.6,11.0,11.0,6.6,2.0 \frac{g}{100 \mathrm{ml} \cdot \mathrm{cm}}\right)$.

\section{Release of Intact DOX}

Two different methods were used to determine that unaltered DOX was released from DOX-PGB.

The first was an HPLC/mass spectrometry analysis where a solution of DOX in DMSO/water (10/90, v/v) and two identical solutions of purified DOX-PCB in DMSO/water $(10 / 90, v / v)$ were prepared. One of the 
Fig. I Synthesis scheme for DOX-PCB

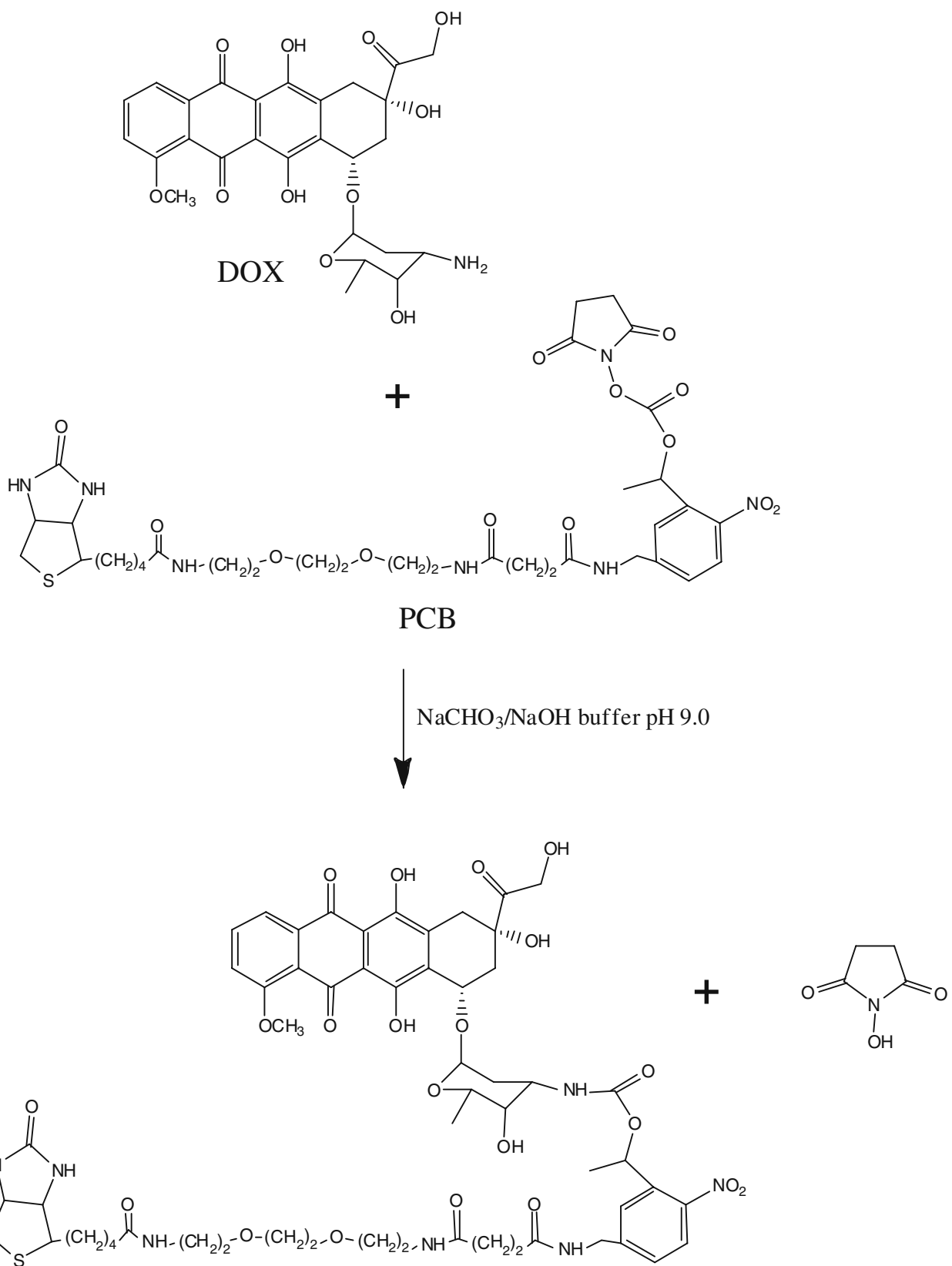

DOX-PCB

DOX-PCB samples was exposed to $120 \mathrm{sec}$ of $2.19 \mathrm{~mW} / \mathrm{cm}^{2} \mathrm{UV}$ created by a source consisting of a mercury arc lamp with a Mercury Short Arc HBO bulb from OSRAM (München, Germany) with a 330-380 nm bandpass filter. The second sample was not exposed to UV. All three samples were then analyzed using HPLC using the same analysis parameters as described above for the characterization.

The second study was a ${ }^{1} \mathrm{H}$ NMR analysis to determine if the free amine of DOX was restored after UV exposure.
The analysis was performed with Watergate water suppression on a JOEL ECA-500 device (Peabody, MA, USA) $500 \mathrm{MHz}$ with 1000 scans for DOX-PCB. The sample consisted of purified DOX-PCB in a solution of DMSO$\mathrm{d}_{6}$ /water $(10 / 90, \mathrm{v} / \mathrm{v})$. An NMR analysis was first run using this sample. The same DOX-PCB sample was then irradiated with $240 \mathrm{~s}$ of UV from the same source as described above. The exposure time was increased to $240 \mathrm{~s}$ to ensure a high percentage of conversion to give a clear signal from the free amine if present. A second NMR 
analysis using the same parameters as before was taken on this UV irradiated sample. A control sample of pure DOX was also dissolved in DMSO- $\mathrm{d}_{6} /$ water $(10 / 90, \mathrm{v} / \mathrm{v})$. More DOX was used than DOX-PCB because DOX was more readily available, and a high signal-to-noise ratio was desired to give the best indication of the chemical shift from the free amine protons. The same NMR analysis was run on this DOX sample using only 16 scans since this sample had a much higher effective concentration.

\section{Cell Uptake and Cellular Localization}

The differences in the intracellular localization between DOX and DOX-PCB were studied using the PTK2 epithelial cell line. This cell line was chosen due to its overall sensitivity to DOX and its natural ability to remain flat during cell division, allowing the mitotic spindle and chromosomes to be readily visible. This feature permitted very clear observation of the interaction of DOX and DOX-PCB with the chromosome material when the nuclear membrane was intact and when it was disassembled at different stages of mitosis. The red fluorescence of DOX and DOX-PCB allowed for easy identification of their intracellular localization. The PTK2 cells were obtained already plated in sealed Rose Chambers incubating in advanced MEM Media with 2\% fetal bovine serum, nonessential amino acids, $110 \mathrm{mg} / \mathrm{L}$ sodium pyruvate, and without penicillin-streptomycin or L-Glutamine from Gibco. A $4 \mathrm{ml}$ solution of $5 \mu \mathrm{M}$ DOX dissolved in the above media was prepared as well as a $4 \mathrm{ml}$ solution of $50 \mu \mathrm{M}$ DOX-PCB. DOX-PCB was used at a higher concentration because its toxicity was already known to be significantly lower than that of free DOX, and it was of interest to determine its intracellular localization. These solutions were injected into the Rose chamber, replacing the $1.8 \mathrm{ml}$ of original media. The cells were allowed to incubate for $2 \mathrm{~h}$ with the drugs before being analyzed using fluorescence microscopy (see subsequent description). After the initial fluorescence analysis, the DOX-PCB incubated cells were then exposed to the UV source for $60 \mathrm{~s}$. The cells were allowed to incubate in this exposed media for $1 \mathrm{~h}$ before being analyzed again by fluorescence microscopy. To determine if the UV exposure affected the cells and their behavior with the DOX-PCB, a sample of DOX-PGB solution was exposed to $60 \mathrm{~s}$ of UV by itself and then was injected into the media of a cell culture chamber.

Images of the live PTK2 cells were obtained using a Zeiss Axiovert 200 M Microscope (Zeiss, Thornwood, NY) with a $63 x$ phase III, NA 1.4 oil immersion objective. An HCred 1 rhodamine filter cube from Chroma (Rockingham, VT, USA) was used for the fluorescent images. All microscope control and imaging utilized the RoboLase system (23).

\section{Cytotoxicity}

The cell line used for the $\mathrm{IC}_{50}$ studies was the human lung cancer cell line A549 purchased from the American Type Culture Collection (ATCG) (Manassas, VA, USA). The cells were grown on sodium pyruvate-free DMEM media containing $4.5 \mathrm{~g} / \mathrm{L}$ of glucose, L-glutamine, 10\% Fetal Bovine Serum, and penicillin-streptomycin. The adherent cells were detached from the expansion flask substrate using Trypsin (.25\% T / $2.21 \mathrm{mM}$ EDTA) and plated onto a 96well plate at a density of $10^{4}$ cells per well with $100 \mu \mathrm{L}$ of media. The cells were allowed to adhere to the bottom of each well overnight.

Experiments were run in two replicates under five different conditions. The first condition was exposure to pure DOX, the second was exposure to DOX-PCB with no UV irradiation, the last three were exposure to DOX-PCB with 2, 20, and $60 \mathrm{~min}$ of UV irradiation from the same source used in the UV stability experiments. The stock solutions of $15.1 \mathrm{mM} \mathrm{DOX}$ and $15.1 \mathrm{mM}$ DOX-PGB in DMSO were diluted to $100 \mu \mathrm{M}$ in media. A $1 / 3$ serial dilution was then performed to create media solutions that ranged from $100 \mu \mathrm{M}$ to $1.7 \mathrm{nM}$. Pure media was used as the control condition. At the $100 \mu \mathrm{M}$ concentration there was no observed precipitation of either DOX or DOX-PCB. The $\mathrm{IC}_{50}$ of DOX-PCB was not reached at $100 \mu \mathrm{M}$ concentration, so a second experiment was run where the maximum concentration of DOX and DOX-PCB was $1000 \mu \mathrm{M}$. The same method of one-third serial dilutions as described above was used for the high concentration $\mathrm{IC}_{50}$ experiment. No significant precipitation of DOXPCB occurred, although there was precipitation of DOX at the $1000 \mu \mathrm{M}$ concentration level.

The media of each well with adherent cells was then replaced with the appropriate premixed media/drug solution, causing minimal disturbance to the cells themselves. The cells were allowed to incubate at $37^{\circ} \mathrm{C}$ for $72 \mathrm{~h}$. After 72 h, an XTT cell viability assay was performed using the In Vitro Toxicology Assay Kit (TOX2) from SigmaAldrich using phenol red free DMEM Media containing 10\% Fetal Bovine Serum and penicillin-streptomycin from Gibco. Absorbance measurements were taken with a Tecan Infinite M200 plate reader (San Jose, CA, USA). The percent viability $v s$. dose curves were generated using the PRISM 4.0 program from GraphPad Software Inc. (La Jolla, CA, USA) with the sigmoidal dose-response (variable slope) curve fit.

\section{UV Stability Experiments}

In order to understand the stability and release characteristics of DOX from the DOX-PCB prodrug upon UV 
exposure, an HPLC/mass spectrometry analysis was performed using an Agilent 1100 Series LC (Santa Clara CA, USA) connected to an Agilent MSD G2445D (Santa Clara CA, USA). The column used was a Supelco Discovery HS C18 $(5 \mathrm{~cm} \times 2.1 \mathrm{~mm}$, with a $3 \mu \mathrm{M}$ bore size) (Bellefonte, PA, USA) and was maintained at a temperature of $20.0^{\circ} \mathrm{C}$. The detection wavelengths were $\lambda_{1} 250 \mathrm{~nm}$ and $\lambda_{2} 400 \mathrm{~nm}$. An $8 \mu \mathrm{L}$ injection was used with a solvent mixture of acetonitrile (ACN) and water with $0.1 \%$ TFA using a gradient of 10-90\% ACN.

First, a standard curve of total ion count peak values for DOX was created using the above protocol and samples of known DOX concentration from $0.01 \mu \mathrm{M}$ to $100 \mu \mathrm{M}$ in $10 \mathrm{X}$ increments in a DMSO/water (20/80, v/v) solution. The peak value of the total ion count for each DOX concentration was collected, and the data was fitted to a Boltzman sigmoidal curve using the program PRISM 4.0 from GraphPad Software Inc. (La Jolla, CA, USA) for the concentration range between 0.01 and $100 \mu \mathrm{M}$.

Duplicate samples of $100 \mu \mathrm{M}$ DOX-PCB in DMSO/ water $(20 / 80, \mathrm{v} / \mathrm{v})$ in $10 \mu \mathrm{L}$ amounts were prepared and then exposed to a $1.8 \mathrm{~mW} / \mathrm{cm}^{2} \mathrm{UV}$ source for different durations of time from 0 to $10 \mathrm{~min}$. The samples were placed in an Optilux Microtest 96-well assay plate (BD Biosciences, San Jose, CA, USA) with opaque black walls and a clear bottom to prevent reflections of UV in the well, ensuring more uniform and repeatable UV exposure to each sample. The cleavage of DOX from DOX-PCB was most sensitive to $350 \mathrm{~nm}$. The peak of the total DOX ion count for each sample was determined using the same HPLC/mass spectrometry protocol as described. These values were then fitted to the standard DOX curve to determine the concentration of the released DOX.

\section{Metabolic Stability Using Human Liver Microsomes}

To test the metabolic stability of the DOX-PCB molecule, a human liver microsome assay was run using the $\mathrm{BD}$ Biosciences (San Jose, CA, USA) NADPH regeneration system. The human liver microsomes were purchased from Sigma and had a protein content of $20 \mathrm{mg} / \mathrm{ml}$. They were pooled from several different human donors. The following protocol was adapted from the NADPH regeneration system suggested protocol.

One assay was run with pure DOX and the other three assays with DOX-PCB. The pure DOX assay acted as the control by looking for the expected metabolite Doxorubicinol, see Fig. 7 d. All reagents were warmed to $37^{\circ} \mathrm{C}$. The assay solution consisted of $80 \mu \mathrm{L}$ of $0.5 \mathrm{M} \mathrm{KPO}_{4}, 20 \mu \mathrm{L}$ of solution A (NADP+and Glc-6-PO4) from the NADPH regeneration system kit, $4 \mu \mathrm{L}$ of solution $\mathrm{B}(\mathrm{G} 6 \mathrm{PDH})$ from the NADPH regeneration system kit, $278 \mu \mathrm{L}$ of $\mathrm{H}_{2} \mathrm{O}$, and $8 \mu \mathrm{L}$ of either an $10 \mathrm{mM}$ solution of DOX or DOX-PCB in
DMSO. The sample of DOX-PCB used here was not purified because the synthesis reaction went nearly to completion with only a residual amount of DOX present. To this solution was added $10 \mu \mathrm{L}$ of the microsomes. The samples were then incubated in a shaker at $37^{\circ} \mathrm{C}$ for $30 \mathrm{~min}$.

At the end of $30 \mathrm{~min}, 400 \mu \mathrm{L}$ of acetonitrile was added to the reaction. The mixture was vortexed for 2 min to stop the reaction of the microsomes and extract DOX and potential DOX-PCB metabolites from the reaction mixture. The mixture was centrifuged until two layers were obtained. The top acetonitrile layer was removed and centrifuged. An HPLC/mass spectrometry analysis was done on the filtered supernatant of the acetonitrile layer using the same analysis protocol for the UV stability study with the detection wavelength at $280 \mathrm{~nm}$.

\section{RESULTS}

\section{Preparation of the Photocleavable Prodrug DOX-PCB}

We have introduced a photosensitive blocking group to reduce the cytotoxicity and reactivity of the chemotherapy drug Doxorubicin with the option of fully regaining these effects upon UV irradiation. Binding the photocleavable blocking group to the active amine within the DOX structure takes advantage of the high reactivity of this group to ensure that the blocking occurs selectively at this controlled location. Attaching certain groups to the active amine itself has been shown to significantly reduce the toxicity of DOX (24). Choosing the correct linking molecules attached to the photocleavable group can reduce the drug's toxicity through steric hindrance and also by specific interactions with various intracellular compartments and membranes. For the design of DOX-PCB, a water soluble photocleavable biotinylated compound (PCB) was chosen which included an ortho-nitrophenyl group for its photo cleavability (25) and a water-soluble glycol spacer arm ending in a biotin group for enhanced membrane interaction. During synthesis, the photocleavable group was protected with an n-hydroxy succunamide group (NHS) which enabled its covalent attachment to the amine group in the DOX structure. The synthetic scheme is shown in Fig. 1.

\section{Characterization of DOX-PCB}

Characterization was performed on DOX-PCB to confirm the structure of the compound proposed in Fig. 1. Highresolution mass spectrometry of DOX-PCB found that it had complexed with $\mathrm{Na}^{+}$. The measured mass of DOX- 
PCB-Na ${ }^{+}$was $1244.4329 \mathrm{~m} / \mathrm{z}$, and the theoretical mass was $1244.4316 \mathrm{~m} / \mathrm{z}$. These two values are well within the $0.4 \%$ calibration error of the analysis and ensure confidence that DOX-PCB had the proposed structure shown in Fig. 1.

An HPLC/mass spectrometry analysis was performed on DOX-PCB. First pure DOX was run and showed a single peak at 22 minutes of elution (peak 1) as seen in Fig. 2a. The analysis of DOX-PCB shown in Fig. $2 \mathrm{~b}$ showed two peaks at 26 and 27 min of elution (peaks 2 and 3). Both peaks had the same characteristic mass of DOX-PCB. These peaks most likely represented two stable diastereoisomeric forms of the compound (26). The biotin portion of

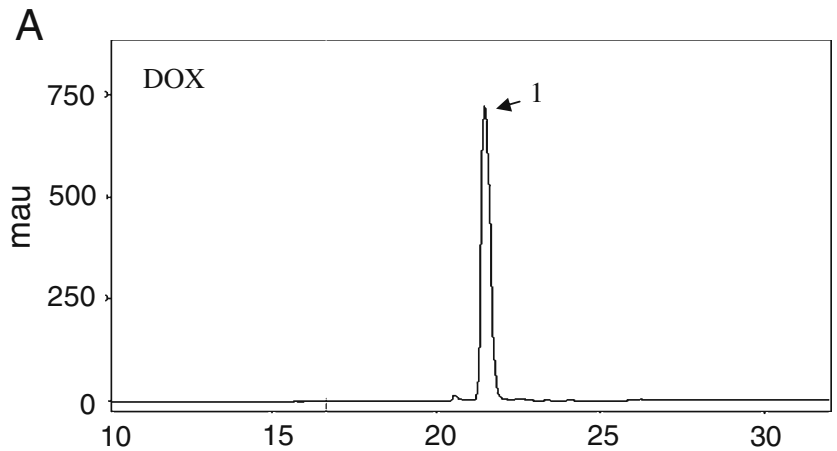

B

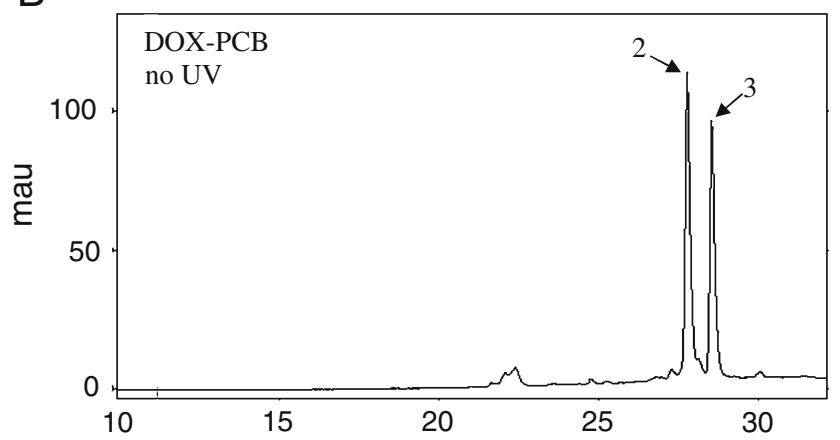

C

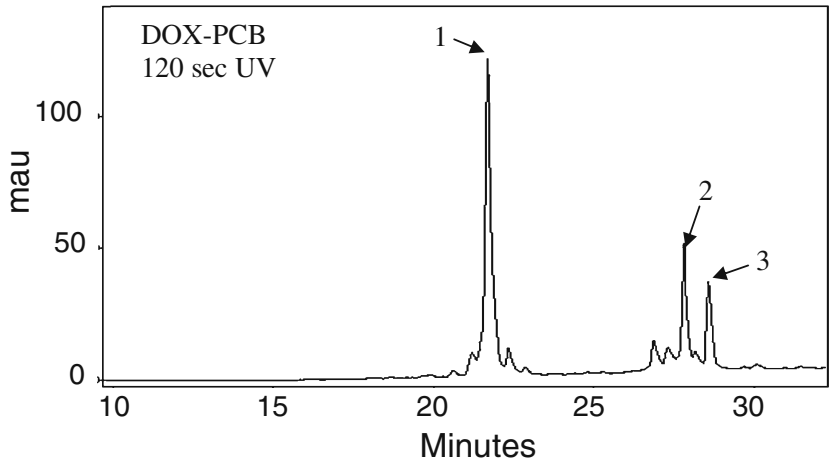

Fig. 2 HPLC analysis of DOX-PCB before and after UV exposure. a Peak I contains pure DOX. b Peaks 2 and 3 both contain DOX-PCB in two diastereoisomeric forms. c The HPLC of the DOX-PCB sample after exposure to UV. The peak I containing DOX appears along with shortened Peaks 2 and 3 containing DOX-PCB. the PCB is chirally homogenous, but the stereo center at the methylene group ortho to the nitro group is a mixture of $\mathrm{R}$ and $\mathrm{S}$ (Ambergen Inc., personal communication). The interaction of $\mathrm{PCB}$ with the hydrophobic portion of the DOX ring structure affects the overall polarity of the two diastereoisomers differently, allowing separation on the C18 column. Both diastereoisomeric forms of DOX-PCB were isolated, and no significant difference in cytotoxicity between them was observed. All the studies described here used DOX-PCB samples that combined the two peaks.

\section{Release of Intact DOX}

It is essential that DOX-PCB release active DOX upon UV exposure so as to obtain the maximum therapeutic effect. Substituents left on the DOX could significantly reduce its toxicity and the overall effectiveness of the prodrug. Releasing free DOX from the DOX-PCB conjugate is based upon the photocleavable characteristic of the nitrophenyl group introduced by Patchornik (25). The mechanism of photolysis has been adapted from established mechanisms and is shown schematically in Fig. 3 (27). Light absorption at $350 \mathrm{~nm}$ causes the electron configurations in the nitrophenyl group to rearrange, inducing the formation of an unstable 5-membered ring with one of the nitro group's oxygen atoms. The destabilization and rearrangement of this ring causes the cleavage, releasing the carbamate group along with DOX. The carbamate undergoes hydrolysis in aqueous conditions, producing $\mathrm{CO}_{2}$ and free DOX.

The release of free active DOX from DOX-PCB upon UV exposure was confirmed by an LC Mass Spectrometry analysis. The HPLC for the purified DOX is shown in Fig. 2a. The mass spectrometry for peak 1 confirms the presence of DOX. The HPLC for purified DOX-PCB with no UV exposure is shown in Fig. 2b. Mass specs for peaks 2 and 3 confirm the presence of DOX-PCB. The HPLC for the DOX-PCB sample that had been exposed to $120 \mathrm{~s}$ of UV is shown in Fig. 2c. The mass spectrometry analysis identified peak 1 as DOX, and peaks 2 and 3 as DOX-PCB.

The release of intact DOX was also confirmed by proton NMR. An NMR of DOX was taken and is shown in Fig. 4a. It displayed a prominent peak at $5.27 \mathrm{ppm}$ attributed to the free amine protons under the solvent conditions of DMSO/ water $(10 / 90, v / v)$. An NMR of a purified DOX-PCB sample should not have a chemical shift peak from the two amine protons due to the carbamate linkage. As expected, the NMR of pure DOX-PCB shown in Fig. 4b demonstrated an absence of the 5.27 ppm peak. This same DOX-PCB sample was then exposed to the UV. The NMR from the sample after the UV exposure is shown in Fig. 4c. The peak at $5.27 \mathrm{ppm}$ was restored, indicating that free DOX was released upon UV exposure. 
Fig. 3 Mechanism of DOX-PCB photolysis and product release with subsequent hydrolysis to produce free DOX and $\mathrm{CO}_{2}$.

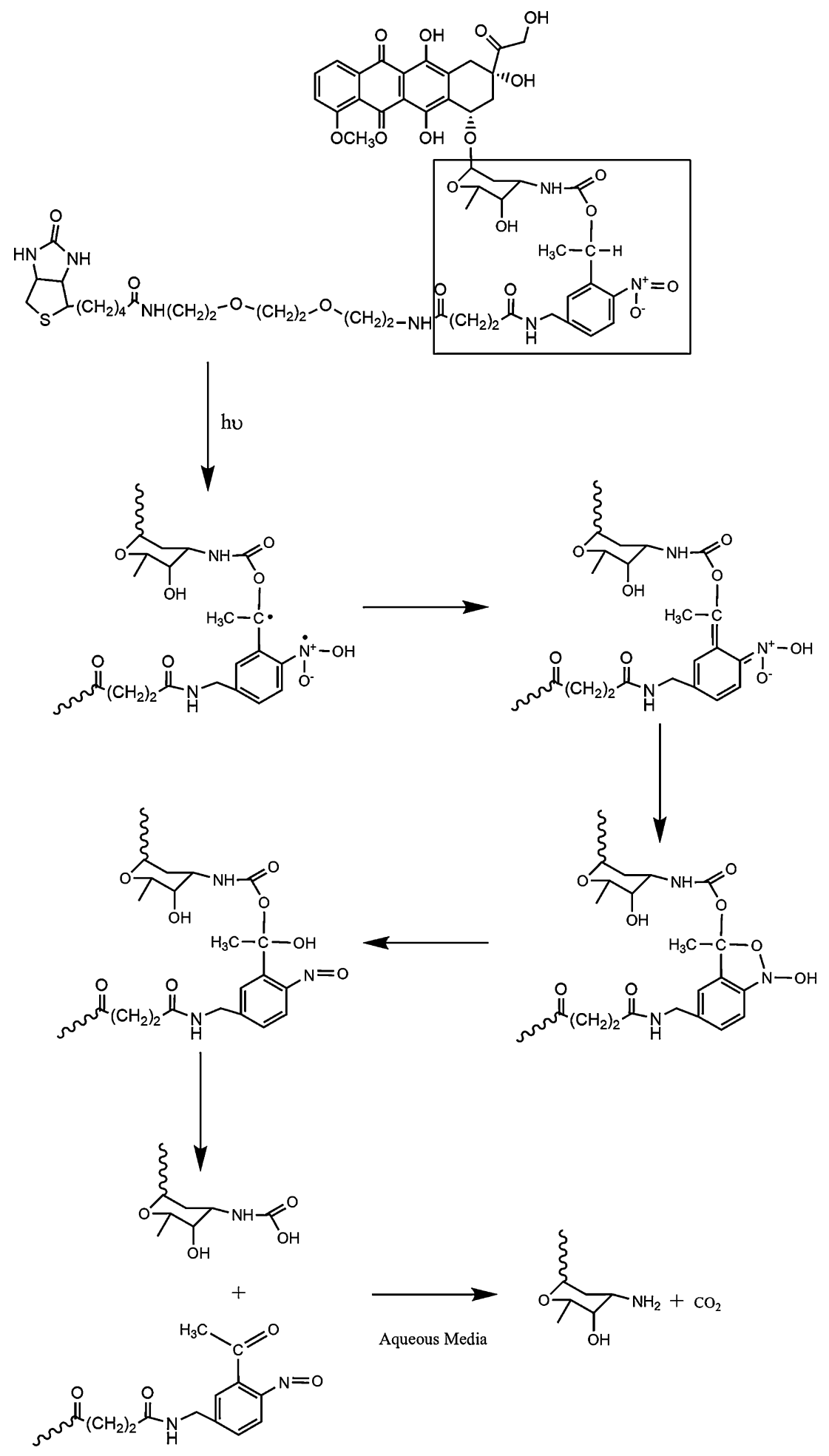

\section{Cell Uptake and Cellular Localization}

The free DOX released from DOX-PCB needs to have the same cellular localization properties as normal free DOX for maximum therapeutic effect. DOX and DOX-PCB are naturally fluorescent, and their localization can be monitored over time in live PTK2 cells using both phase contrast images (top row in Fig. 5) and fluorescent images of the same field (bottom row of Fig. 5). The intracellular localization of DOX was distinct from that of DOX-PCB. Within hours of exposure, DOX entered the cells and was concentrated almost exclusively in the nucleus as pointed out by the 

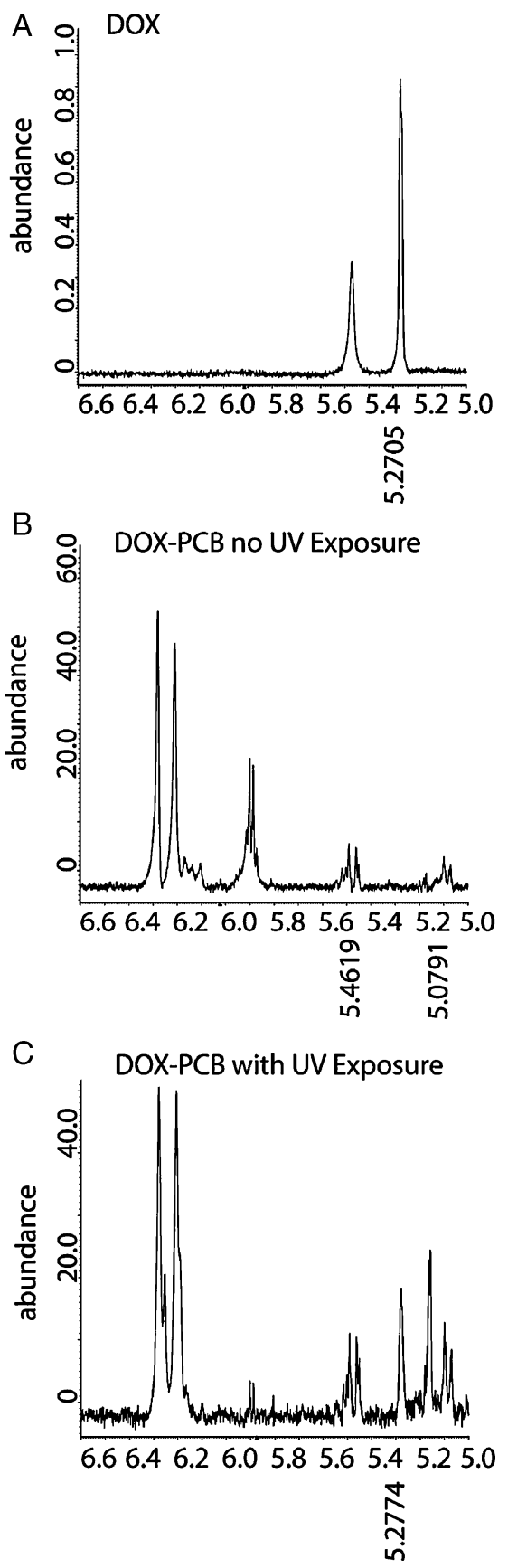

$\mathrm{X}$ : parts per Million : $1 \mathrm{H}$

Fig. $4 \mathrm{NMR}$ analysis of the chemical shift for free DOX, pure DOXPCB, and DOX-PCB after UV exposure. a The NMR of DOX showed the chemical shift for the two amine protons to be at $5.27 \mathrm{ppm}$ (DMSO/ water, 10/90, v/v). b This peak was absent in a sample of pure DOXPCB. c After exposure to UV, the DOX-PCB sample had a restoration of the $5.27 \mathrm{ppm}$ peak indicating the release of free DOX.

arrows in Fig. 5a and b. The top cell of Fig. 5a was not undergoing mitosis, and DOX penetrated the nuclear membrane and associated with the chromatin most likely through a DNA intercalation mechanism (28). It can be seen that the cell in the middle of Fig. 5a was undergoing an abnormal mitosis. The nuclear membrane was degraded, and DOX strongly localized to the exposed chromosomes. The abnormal-appearing mitosis was most likely caused by the interference of DOX with the DNA replication mechanisms. There were very few observations of cells undergoing mitosis, all of which appeared abnormal.

DOX-PCB also entered the cell quickly. However, it did not concentrate in the nucleus as pointed out by the arrows in Fig. 5c and d. DOX-PGB appeared to preferentially accumulate within the cell in regions around the nucleus. Future studies using colocalization staining techniques will help identify which cellular structures DOX-PCB interacts with. The right-most cell is shown undergoing a normalappearing mitosis where the nuclear membrane has disassembled. It is clear that DOX-PCB had been excluded from the mitotic spindle and the associated chromosomes. The DOX-PCB concentration was 10 times higher than DOX, and the cells seemed much healthier and appeared to be undergoing normal mitosis.

When the same DOX-PCB chamber of cells from Fig. 5c and $\mathrm{d}$ was exposed to $\mathrm{UV}$, the red fluorescence accumulated in the nucleus $1 \mathrm{~h}$ after exposure as pointed out in Fig. 5e and $f$. The free DOX that was released from DOX-PCB appeared to be behaving similar to free DOX with its ability to pass through the nuclear membrane and bind to the chromatin. The DOX-PCB that was not photolysed by the UV remained outside the nucleus. These cells appeared to have pyknotic nuclei with a darkened nuclear envelope and also appeared to have highly granulated cytoplasms, both features of cell death. The UV dose received by the cells themselves did not influence their interaction with DOXPBC. This was evidenced by the fact that control cells which were not exposed to UV, but were incubated with a DOXPCB sample that was separately irradiated by UV, exhibited the same behavior (data not shown).

\section{Cytotoxicity}

For the prodrug effect to be pharmacologically useful, the DOX-PCB needs to show a greatly reduced toxicity compared to that of DOX. The results of the cell proliferation assay on A549 human lung cancer cells are shown in Fig. 6a. The $\mathrm{IC}_{50}$ of the DOX sample was $1.2 \mu \mathrm{M}$. The $\mathrm{IC}_{50}$ of DOX-PCB was over 200 times higher at $250 \mu \mathrm{M}$, indicating a significant prodrug effect. The large prodrug effect of DOX-PCB makes it a good candidate to reduce the undesirable systemic side effects of DOX, especially the cardiotoxicity. The observed lack of DOX-PGB interaction with DNA helps to explain the significant reduction in the cytotoxicity of DOX-PGB over that of DOX.

For the prodrug effect to be truly functional, the toxicity of the DOX-PCB sample needs to increase upon UV exposure and release fully active DOX with 

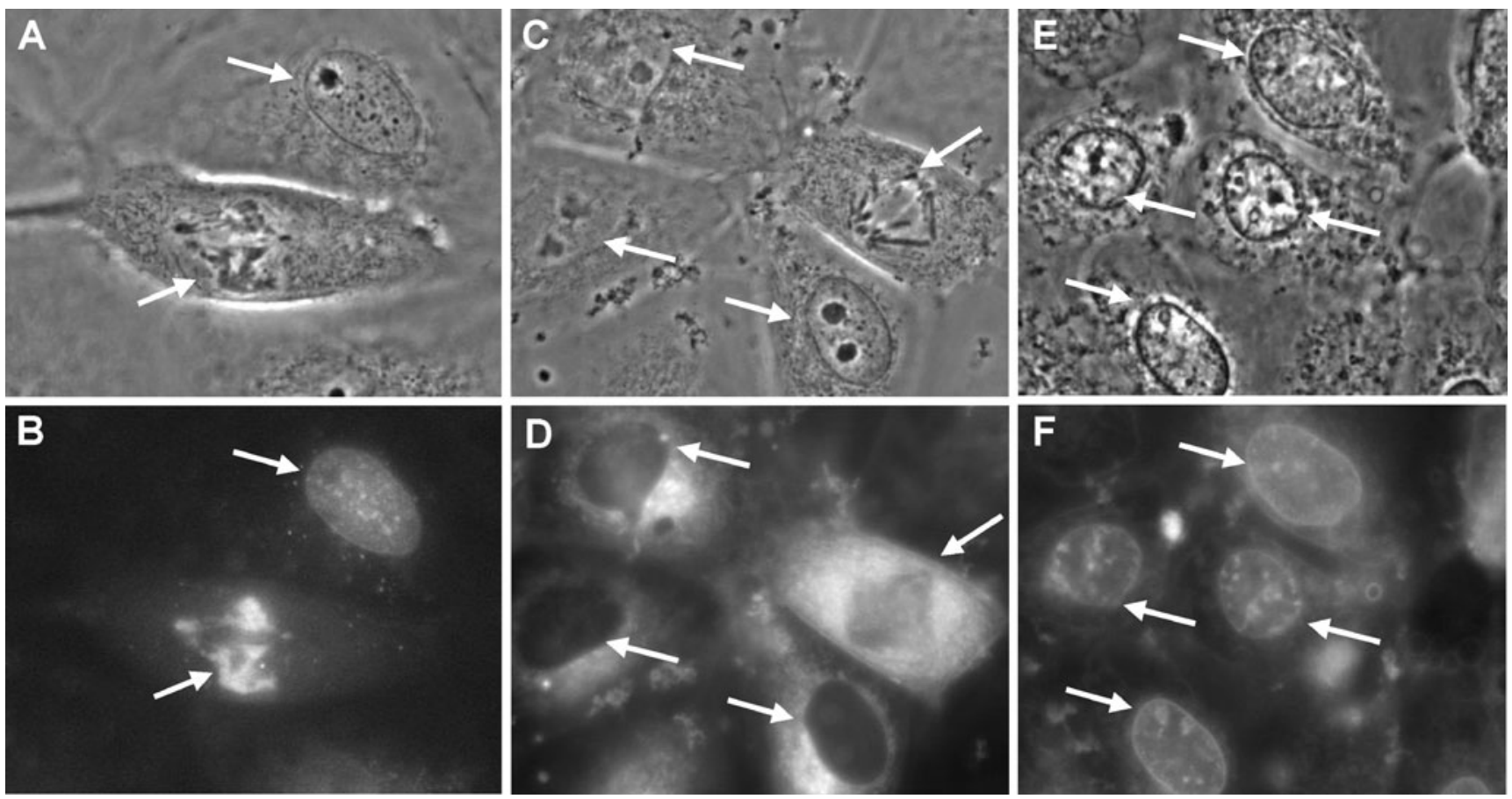

Fig. 5 Microscopy images of DOX and DOX-PCB localization within PTK2 kidney epithelial cells. Panels A, C, and E (top row) are phase contrast images of the cells. Panels B, D, and F (bottom row) are fluorescent images of the same field of view as the images directly above. (a, b) DOX strongly associated with the chromosomes as pointed out by the arrows. (c, d) DOX-PCB entered the cells but did not associate with the chromosomes as pointed out by the arrows. (e, f) When exposed to UV, free DOX was released from DOX-PCB and the chromosomes began to fluoresce as seen pointed out by the arrows.

well-characterized potent bioactivity. Fig. 6b shows a table of $\mathrm{IC}_{50}$ values for DOX-PCB samples exposed to the UV for increasing amounts of time. The DOX-PCB samples dissolved in media were exposed to the UV separately and then incubated with the cells, so the cells were never exposed to UV. As exposure time of the media increased, the $\mathrm{IC}_{50}$ decreased, approaching that of free
DOX due to the increasing amounts of free DOX that were released from the DOX-PCB.

\section{UV Stability Experiments}

The release characteristics of DOX from the DOX-PCB prodrug had a linear relationship $\left(\mathrm{R}^{2}=0.99\right)$ with the

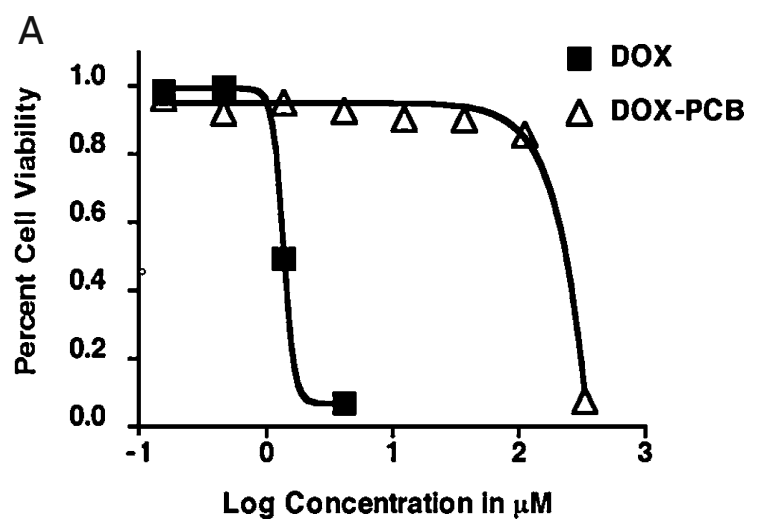

B

\begin{tabular}{cc}
\hline $\begin{array}{c}\text { UV Exposure time } \\
\text { min }\end{array}$ & $\begin{array}{c}\mathrm{IC}_{50} \text { Values } \\
\mu \mathrm{M}\end{array}$ \\
\hline 0 & 250 \\
2 & 100 \\
20 & 5 \\
60 & 3.5 \\
DOX & 1.2 \\
\hline
\end{tabular}

Fig. 6 Cell viability results from DOX, DOX-PCB, and DOX-PCB exposed to increasing amounts of UV. a Cell viability curve for A549 human lung cancer cells exposed to DOX and DOX-PCB with no UV exposure. The IC 50 for DOX was I.2 $\mu$ M. The IC 50 of DOX-PCB with no

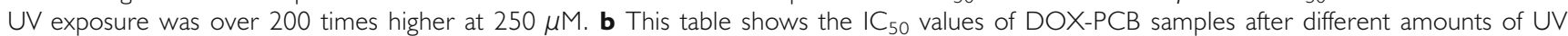
exposure. The DOX-PCB samples were dissolved in media, exposed to the UV, and then incubated with the cells, so the cells were not exposed to UV. As UV exposure time increased, the $I_{50}$ value decreased approaching that of pure DOX due to the higher doses of free DOX released from the DOX-PCB. 
amount of UV exposure (data not shown). After exposure to the $1.8 \mathrm{~mW} / \mathrm{cm}^{2} \mathrm{UV}$ source for $10 \mathrm{~min}, 18 \%$ of the total DOX-PCB content in a $100-\mu \mathrm{m}$ sample was converted to DOX. The release rate of intact DOX was $1.8 \mu \mathrm{M} / \mathrm{min}$.

\section{Metabolic Stability Using Human Liver Microsomes}

To maintain control over the spatial location of DOX-PCB activation, it is important that the only significant pathway of activation be from UV exposure and not from normal metabolic activity, especially from the liver. Incubation of DOX-PCB with human liver microsomes indicates that the compound is quite resistant to metabolic breakdown. Fig. 7a shows the HPLC results of the LC Mass analysis of DOXPCB after incubation with the human liver microsomes. Peak 1 contains DOX-Cl, a residue most likely left over from the synthesis. Peak 2 contains DOX-Na, which is again most likely a residue from the synthesis. Peak 3 contains DOXPCB. Peak 4 contains DOX-PCB, most likely the second isomer, as well as a mass spectrometry peak at $579 \mathrm{~m} / \mathrm{z}$ with the proposed structure shown in Fig. 7b. This $579 \mathrm{~m} / \mathrm{z}$ metabolite is not the DOX-Cl complex because it shows up in the 10.2-10.5 min peak on the HPLC, whereas DOX-Na and DOX-Cl showed up well before that. The precursor to the $579 \mathrm{~m} / \mathrm{z}$ metabolite was found in the first of three replicates of this experiment with a mass of $587 \mathrm{~m} / \mathrm{z}$ with the proposed structure shown in Fig. 7c. The hydrolyzing enzymes in the liver microsomes most likely cleaved the DOX-PCB molecule producing the unstable $587 \mathrm{~m} / \mathrm{z}$ fragment which was then fully reduced to the $579 \mathrm{~m} / \mathrm{z}$ metabolite, much like DOX is reduced to Doxorubicinol shown in Fig. 7d. The main $579 \mathrm{~m} / \mathrm{z}$ metabolite was produced in relatively small quantities and has a substituent on the active amine. Although it is unknown what effect this particular substituent will have on the toxicity of this metabolite, it has been demonstrated that similar substituents significantly reduce the toxicity of DOX (24).

The product of the microsome incubation of DOX was Doxorubicinol. Doxorubicinol has previously been established as the major metabolite of DOX with the structure shown in Fig. $7 d(29,30)$. This served as a positive control for the microsome protocol.
A

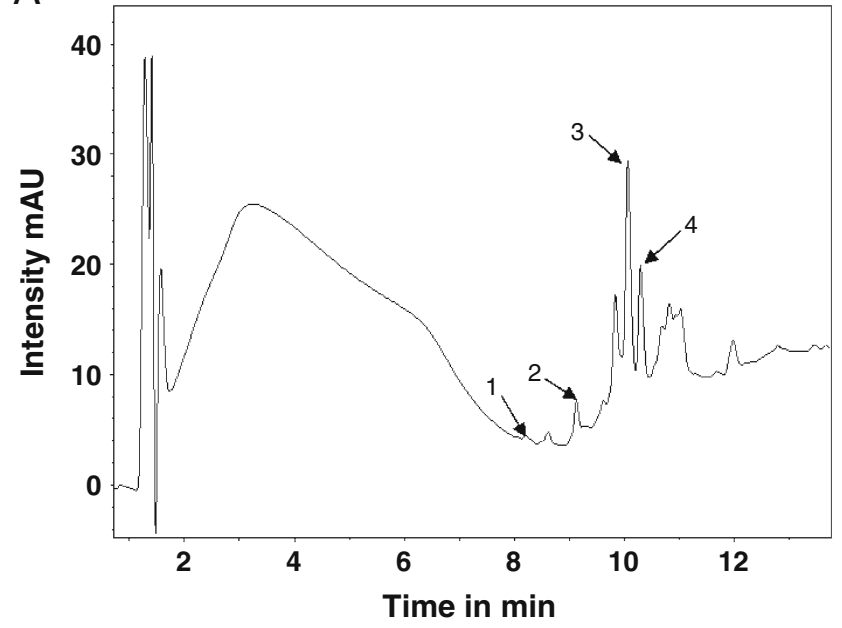

C

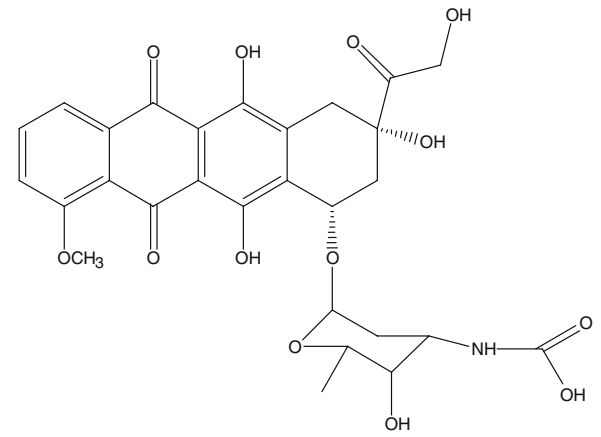

Exact Mass: 587.16
B<smiles>COc1cccc2c1C(O)c1c(O)c3c(c(O)c1C2O)C[C@@](O)(C(O)CO)C[C@@H]3OC1CC(NCO)C(O)C(C)O1</smiles>

Exact Mass: $\mathbf{5 7 9 . 2 3}$<smiles>COc1cccc2c1C(=O)c1c(O)c3c(c(O)c1C2=O)C[C@@](O)(C(O)CO)C[C@@H]3OC1CC(C)C(O)C(N)C1</smiles>

Exact Mass: $\mathbf{5 4 5 . 1 9}$

Fig. 7 LC mass analysis of DOX-PCB incubated with human liver microsomes and the proposed structures of the resulting metabolites. a HPLC from the LC mass analysis of the human liver microsome experiments. b Proposed structure for the $579 \mathrm{~m} / \mathrm{z}$ DOX-PCB metabolite. c Proposed structure for the $587 \mathrm{~m} / \mathrm{z}$ DOX-PCB metabolite that might be the precursor for the $579 \mathrm{~m} / \mathrm{z}$ metabolite. d Structure of Doxorubicinol. 


\section{DISCUSSION}

In this paper, we have developed a new photocleavable prodrug for cancer therapy by incorporating the active chemotherapy agent DOX with the photocleavable protecting group PCB to form the adduct DOX-PCB. It has been shown that DOX-PCB is over 200 times less toxic than DOX, and upon irradiation active DOX is released. With the use of small light-emitting diodes or fiber optics to deliver controlled localized doses of $350 \mathrm{~nm}$ light to desired tissue regions, it should be possible to administer a systemic dose of the prodrug and release active DOX only within the tumor region. The prodrug has the potential to enter cells in its caged form without being metabolized to a pharmacologically active form. A systemic administration of the prodrug would allow it to enter cells and remain in the region of interest even after the freely circulating prodrug has been cleared from the system. It is important to note that a control experiment was run where the A549 cells were exposed to the $1.8 \mathrm{~mW} / \mathrm{cm}^{2} \mathrm{UV}$ light source alone with no reduction in cell viability compared to control cells after $20 \mathrm{~min}$ of exposure. After $60 \mathrm{~min}$ of exposure, $90 \%$ viability was observed, which might have been contributed by the 334 emission peak from the Mercury Arc lamp leaking through the lower end of the 330-380 nm bandpass filter (31). This shows that the main effect of cell viability reduction would be from the released DOX at the 60-min exposure limit.

The incorporation of the biotin moiety is intended to help increase the clearance rate of the freely circulating DOX-PCB just before UV exposure. Free DOX-PGB in the tumor bloodstream at the time of UV exposure would release free DOX into the circulation. Only DOX-PCB that is present inside the tumor cells should be activated. The biotin on DOX-PCB allows for use of a clearing agent, such as modified versions of biotin-galactose-human serum albumin (32) or biotin-LC-NM-(GalNac) ${ }_{16}$ (33), where the biotin would be replaced with streptavidin. These clearing agents very effectively reduce the circulating blood concentration of the target molecule and are designed to use the galactose residue to trigger uptake by galactose receptors in hepatocytes where the entire complex is metabolized. The human microsome experiments indicate that the DOX-PCB drug will remain intact through that process. The biotin end of the prodrug will also help increase the clearance rate of the $\mathrm{PCB}$ fragment created by the UV exposure.

The presence of the biotin on DOX-PCB should not cause undesirable accumulation or protein binding in vivo as has been shown with a compound comprised of biotin bound to a chelated radioisotope (32). The biotin present on this chelated radioisotope did not cause significant accumulation in any particular tissue, including the liver. The compound was rapidly cleared from the body through the renal system.
The use of photocleavable prodrugs offers an exciting new avenue of investigation in reducing the systemic side effects of chemotherapy drugs and other therapeutic agents while increasing their effectiveness in the desired tissue region. Future work will focus on extensive in vivo characterization, including in vivo stability of DOX-PCB, as well as its possible therapeutic effectiveness.

\section{CONCLUSION}

One strategy to reduce the toxic side effects of chemotherapy agents is to make prodrugs by chemically modifying the agents so as to reduce their overall toxicity, but also allow them to be activated back to a therapeutic state in the tumor tissue. One of the challenges is to differentiate the tumor from the healthy tissue in order to effectively trigger the prodrug only in the tumor. The triggers of many prodrug designs rely on inherent biochemical differences between the tumor and healthy tissue. These differences can be very subtle, and often healthy tissues within the body will have the same biochemistry as the tumor. To avoid the undesirable activation of the prodrug in these untargeted tissues, an artificial difference can be created within the tumor tissue from a source external to the body. The trigger considered here is UV radiation at $350 \mathrm{~nm}$. This is at the very low energy end of the UV spectrum with good tissue penetration and little interaction with DNA and protein. A new prodrug of doxorubicin (DOX-PCB) is described that can be converted to the active drug upon exposure to UV at $350 \mathrm{~nm}$. It was formed by conjugating a photocleavable blocking group to the sugar amine group of DOX. DOX$\mathrm{PCB}$ was shown to have a significant reduction in toxicity over free DOX with a 200 -fold lower $\mathrm{IC}_{50}$ in cell viability tests. One of the possible reasons for the lower toxicity is that the PCB portion blocks DOX-PCB from intercalating with DNA, which is a main mode of action for DOX. DOX$\mathrm{PCB}$ was shown to be stable in a human microsome experiment, indicating that DOX will not be released in a significant amount due to metabolic conversion. DOX-PCB was shown to release biologically active doxorubicin upon exposure to UV at $350 \mathrm{~nm}$, increasing the cytotoxicity of the entire sample. It is possible to deliver UV only to the tumor region using UV fiber optics or implantable UV LEDs. This could cause the activation of systemically administered DOX-PCB only in the tumor, thereby reducing systemic side effects.

\section{ACKNOWLEDGEMENTS}

The authors are grateful for the support of Anthony Mrse, Norman Baker, Yongxuan Su, Dmitri Simberg, Christina $\mathrm{Wu}$, Dominic Yee, Howard Cottam, and Lianglin Zhang in the collection and interpretation of the data. The authors 
also wish to thank Ambergen Inc., for clarification of the structural details of the PCB diastereoisomers. The study was supported by the National Cancer Institute grant \# 5 U54 CA1 19335.

Open Access This article is distributed under the terms of the Creative Commons Attribution Noncommercial License which permits any noncommercial use, distribution, and reproduction in any medium, provided the original author(s) and source are credited.

\section{REFERENCES}

1. Gvetkovic RS, Scott LJ. Dexrazoxane a review of its use for cardioprotection during anthracycline chemotherapy. Drugs. 2005;65:1005-24.

2. Pawan NI, Singal K. Doxorubicin-induced cardiomyopathy. N Engl J Med. 1998;339:900-5.

3. Mushlin P, Olson RD. Doxorubicin cardiotoxicity: analysis of prevailing hypotheses. FASEB J. 1990;4:3076-86.

4. Menna P, Minotti G, Salvatorelli E, Cairo G, Gianni L. Anthracyclines: molecular advances and pharmacologic developments in antitumor activity and cardiotoxicity. Pharmacol Rev. 2004;56:185-229.

5. Denny WA. Prodrug strategies in cancer therapy. Eur J Med Chem. 2001;36:577-95.

6. Denny WA. Tumor-activated prodrugs - a new approach to cancer therapy. Cancer Investig. 2004;22:604-19.

7. Lutz MN, Tietze F, Mollers T, Fischer R, Glusenkamp K-H, Rajewsky MF, et al. Proton-mediated liberation of aldophosphamide from a nontoxic prodrug: a strategy for tumor-selective activation of cytocidal drugs. Cancer Res. 1989;49:4179-84.

8. Wilson WR, Brown JM. Exploiting tumor hypoxia in cancer treatment. Nat Rev Cancer. 2004;4:437-47.

9. Breistol HRHK, Berger DP, Langdon SP, Fiebig HH, Fodstad O. The antitumour activity of the prodrug N-l-leucyl-doxorubicin and its parent compound doxorubicin in human tumour xenografts. Eur J Cancer. 1998;34:1602-6.

10. Gopin A, Ebner S, Attali B, Shabat D. Enzymatic Activation of second-generation dendritic prodrugs: Conjugation of selfimmolative dendrimers with PEG via click chemistry. Bioconjug Chem. 2006;17:1432-40.

11. Shamis M, Lode HN, Shabat D. Bioactivation of self-immolative dendritic prodrugs by catalytic antibody 38C2. JACS. 2004;126:1726-31.

12. Wrasidlo WA, Mueller BM, Reisfeldt RA. Antibody conjugates with morpholinodoxorubicin and acid-cleavable linkers. Bioconjug Chem. 1990;1:325-30.

13. Shoemaker RH, Scudiere DA, Paull KD, Monks A, Tierney S, Nofziger TH, et al. A distinct "side population" of cells with high drug efflux capacity in human tumor cells. PNAS. 2004;101:1422833.

14. Tachi Y, Shibamoto Y, Tanabe K, Hatta H, Nishimoto S-I. In vitro and in viwo evaluation of novel antitumor prodrugs of 5-fluoro-2 deoxyuridine activated by hypoxic irradiation. Int J Radiat Oncol Biol Phys. 2004;58:397-402.
15. Noguchi M, Mariusz S, Halan P, Shun H, Tooru K, Yoshio H, et al. Development of novel water-soluble photocleavable protective group and its application for design of photoresponsive paclitaxel prodrugs. Bioorg Med Chem. 2008;16:5389-97.

16. Dachs GU, Greco O. Gene directed enzyme/prodrug therapy of cancer: historical appraisal and future prospectives. J Cell Physiol. 2001;187:22-36.

17. Sharma SK, Napier MP, Springer CJ, Bagshawe KD, Green AJ, Martin J, et al. Antibody-directed enzyme prodrug therapy: efficacy and mechanism of action in colorectal carcinoma. Clin Cancer Res. 2000;6:765-72.

18. Hobson DA, Pandori MW, Olejnik J, Krzymanska-Olejnik E, Rothschild KJ, Palmer AA, et al. Photochemical control of the infectivity of adenoviral vectors using a novel photocleavable biotinylation reagent. Chem Biol. 2002;9:567-73.

19. Amit B, Zehavi U, Patchornik A. Photosensitive protecting groups of amino sugars and their use in glycoside synthesis. 2-nitrobenzyloxycarbonylamino and 6-nitroveratryloxycarbonylamino derivatives. J Org Chem. 1974;39:192-6.

20. Elisseeff KAJ, Sims D, McIntosh W, Randolph M, Langer R. Transdermal photopolymerization for minimally invasive implantation. Proc Natl Acad Sci. 1999;96:3104-7.

21. Celmer EJ, Yang Y, Koutcher JA, Alfano RR. UV reflectance spectroscopy probes DNA and protein changes in human breast tissues. J Clin Laser Med Surg. 2001;19:35-9.

22. Sutherland J, Griffin K. Absorption spectrum of DNA for wavelengths greater than $300 \mathrm{~nm}$. Radiat Res. 1981;86:399-410.

23. Botvinick E, Berns M. Internet-based robotic laser scissors and tweezers microscopy. Microsc Res Tech. 2005;68:65-74.

24. Lau DHM, Lewis AD, Duran GE, Wolf CR, Sikic BI. Role of cytochrome P-450 from the human CYP3A gene family in the potentiation of morpholino doxorubicin by human liver microsomes. Cancer Res. 1992;52:4379-84.

25. Patchornik A, Amit B, Woodward RB. Photosensitive protecting groups. JACS 1970;6333-5.

26. Olejnik J, Sonar S, Krzymanska-Olejnik E, Rothschild K. Photocleavable biotin derivatives: a versatile approach for the isolation of biomolecules. Proc Natl Acad Sci USA. 1995;92:7590-4.

27. Schupp WKWH, Schnabel W. Mechanistic studies of the photorearrangement of o-nitrobenzyl esters. J Photochem. 1987;36:85-97.

28. Wang AH-J, Quigley GJ, Ughetto G, Van Der Marel G, Van Boom JH, Rich A. Molecular structure of an anticancer drugDNA complex: daunomycin plus d(CpGpTpApCpG). Proc Nati Acad Sc USA. 1980;77:7204-8.

29. Lum BL, Chin DL, Sikic BI. Rapid determination of PEGylated liposomal doxorubicin and its major metabolite in human plasma by ultraviolet - visible high-performance liquid chromatography. J Chromatogr B. 2002;779:259-69.

30. Bachur NR, Takanashi S. Adriamycin metabolism in man evidence from urinary metabolites. Drug Metab Dispos. 1975;4:79-87.

31. Peters D. A comparison of mercury arc lamp and laser illumination for flow cytometers. J Histochem Cytochem. 1979;27:241-5.

32. Reno JM, Axworthy DB, Hylarides MD, Mallett RW, Theodore LJ, Gustavson LM, et al. Cure of human carcinoma xenografts by a single dose of pretargeted yttrium-90 with negligible toxicity. PNAS. 2000;97:1802-7.

33. Breitz HB, Weiden PL. Pretargeted radioimmunotherapy (PRIT ${ }^{\mathrm{TM}}$ ) for treatment of non-Hodgkin's lymphoma (NHL). Crit Rev Oncol Hematol. 2001;40:37-51. 